\title{
Analysis of structure indicators influencing 3-h and 6-h compliance with the surviving sepsis campaign guidelines in China: a systematic review
}

Lu Wang ${ }^{1,2+}$, Xudong Ma ${ }^{3+}$, Huaiwu He ${ }^{1,2}$, Longxiang Su ${ }^{1,2}$, Yanhong Guo ${ }^{3}$, Guangliang Shan ${ }^{4}$, Xiang Zhou Z $^{1,2^{*}} \mathbb{0}$, Dawei Liu ${ }^{1,2^{*}}$ and Yun Long ${ }^{1,2}$ China National Critical Care Quality Control Center Group

\begin{abstract}
Background: Compliance with the surviving sepsis campaign (SSC) guidelines $\left(\mathrm{C}_{\mathrm{ss}}\right)$ is a key factor affecting the effects of sepsis treatment. We designed this study to investigate the relationships of the structure indicators of ICU on 3 and 6-h $C_{\text {ssc }}$ in China.

Methods: A total of 1854 hospitals were enrolled in a survey, led by the China National Critical Care Quality Control Center (China-NCCQC) from January 1, 2018, through December 31, 2018. We investigated the 1854 hospitals' 3 and 6-h $C_{\text {ssc }}$ including compliance with each specific measure of the 3-h and 6-h SSC bundles. We also investigated the actual level of the structure indicators of ICU, released by China-NCCQC in 2015. The outcomes were in adherence with the SSC guidelines (2016). Monitoring indicators included 3 and 6-h $C_{\text {ssc }}$.

Results: In the subgroup, the rate of broad-spectrum antibiotic therapy was the highest, and the rate of CVP and $\mathrm{ScvO} 2$ measurement was the lowest among the items of 3 and $6-\mathrm{h} C_{\text {ssc }}$. Structure indicators related to 3 and 6 -h $C_{\text {ssc }}$ include the predicted mortality rate and the standardized mortality ratio (SMR). The relationships between 3 and 6-h $C_{\text {ssc }}$ and the proportion of ICU in total inpatient bed occupancy, the proportion of acute physiology and chronic health evaluation (APACHE) II score $\geq 15$ in all ICU patients were uncertain. There was no relationship of 3 and 6 -h $C_{\text {ssc }}$ with the proportion of ICU patients among total inpatients.
\end{abstract}

Conclusions: Structure indicators influencing 3 and $6-\mathrm{h} C_{\text {ssc }}$ in China are the predicted mortality rate and the standardized mortality rate.

Keywords: Structure indicator, ICU, Compliance, Surviving sepsis campaign guidelines, China

*Correspondence: zx_pumc@126.com; daweiliu_pucmhicu@163.com ${ }^{\dagger}$ Lu Wang and Xudong Ma contributed equally to this work

${ }^{1}$ Department of Critical Care Medicine, Peking Union Medical College and Chinese Academy of Medical Sciences, Peking Union Medical College Hospital, Beijing 100730, China

Full list of author information is available at the end of the article

\section{Background}

Sepsis 3.0 is defined as downregulation of host response after infection and the occurrence of life-threatening organ dysfunction $[1,2]$. The overall global burden of sepsis has increased over the past several decades. Recent studies have shown that the mortality from sepsis can be reduced by compliance with the surviving sepsis campaign (SSC) guidelines $\left(C_{\mathrm{ssc}}\right)$ [3-5]. However, the $C_{\mathrm{ssc}}$ in clinical work is only approximately $30-60 \%$ [6].

(c) The Author(s) 2021. This article is licensed under a Creative Commons Attribution 4.0 International License, which permits use, sharing, adaptation, distribution and reproduction in any medium or format, as long as you give appropriate credit to the original author(s) and the source, provide a link to the Creative Commons licence, and indicate if changes were made. The images or other third party material in this article are included in the article's Creative Commons licence, unless indicated otherwise in a credit line to the material. If material is not included in the article's Creative Commons licence and your intended use is not permitted by statutory regulation or exceeds the permitted use, you will need to obtain permission directly from the copyright holder. To view a copy of this licence, visit http://creativeco mmons.org/licenses/by/4.0/. The Creative Commons Public Domain Dedication waiver (http://creativecommons.org/publicdomain/ zero/1.0/) applies to the data made available in this article, unless otherwise stated in a credit line to the data. 
The structure indicators of intensive care unit (ICU) reflect whether the resource allocation of the ICU meets the requirements. At present, there are few studies on relationship between structure indicators and the $C_{\mathrm{ssc}}$ [7]. We assume that the structure indicators will have an important impact on the $C_{\text {ssc }}$. Therefore, the aims of this study were the following: 1 . to investigate the $C_{\text {ssc }}$ and structure indicators of ICU in China; and 2. determine the relationships between the $C_{\mathrm{ssc}}$ and structure indicators of ICU

\section{Methods Hospitals}

The total number of secondary and tertiary hospitals registered was 7525 across the country in 2018. China National Critical Care Quality Control Center (ChinaNCCQC) collected detailed data regarding quality control indicators through the database of the National Clinical Improvement System (https://icuqc.console.clini fy.cn/dataMonitoring). The data were collected between January 1, 2018 and December 31, 2018. Hospitals with patients of septic shock admitted in ICUs $<20$ /year and incomplete data were excluded from this study. There were only 6 private specialized hospitals, including 4 tertiary hospitals and 2 secondary hospitals, so they were not included in this survey. At last, 1854 hospitals in China were involved. All of the information from participating hospitals is listed in Table 1 and Additional file 1. The 3 and 6-h $C_{\text {ssc }}$ are listed in Table 2 and Additional file 2: Figures S1-S4 and Additional file 3: Figures S1-S4.

\section{Study design}

In this study, the structure indicators of ICU were evaluated according to the National Clinical Quality Control
Indicators for Critical Care Medicine (2015 Edition) released by the China-NCCQC. Monitoring indicators included the proportion of ICU patients among total inpatients, the proportion of ICU patients out of total inpatient bed occupancy, the proportion of APACHE II scores $\geq 15$ in all ICU patients, the predicted mortality rate and the standardized mortality ratio. Each indicator is divided into 4 grades according to the implementation. Each $25 \%$ from bad to good is divided into the lowest group, the lower group, the higher group, and the highest group.

The primary end points were the 3 and $6-\mathrm{h} C_{\mathrm{ssc}}$. Monitoring indicators included 3 -h $C_{\mathrm{ssc}}$ (1. Completion of lactate concentration was determined, 2. Completion of appropriate routine microbiologic cultures [including blood] obtained before starting antimicrobial therapy, 3 . Completion of empiric broad-spectrum therapy, 4. Completion of resuscitation with $30 \mathrm{~mL} / \mathrm{kg}$ crystal liquid) and 6-h $C_{\text {ssc }}$ [1. Completion of repeated measurement of lactate levels in patients with initial hyperlactatemia, 2 . Completion of resuscitation with vasopressor in patients with mean arterial pressure (MAP) $\leq 65 \mathrm{mmHg}$ after fluid resuscitation, 3 . Completion of central venous pressure $(\mathrm{CVP})$ and central venous oxygen saturation $\left(\mathrm{ScvO}_{2}\right)$ measured in patients with lactate $\geq 4 \mathrm{mmol} / \mathrm{L}]$.

According to the above levels, we investigated the relationships of the structure indicators of ICU on 3 and 6-h $C_{\text {ssc }}$ in patients with sepsis in China.

The study was conducted in accordance with the Declaration of Helsinki (as revised in 2013). The trial protocol was approved by the Central Institutional Review Board at Peking Union Medical College Hospital (NO.: $\mathrm{S}-\mathrm{K} 1297)$ and individual consent for this retrospective analysis was waived. The authors are accountable for all

Table 1 Basic information of different types of hospitals

\begin{tabular}{|c|c|c|c|c|c|c|c|c|c|}
\hline & Hospitals & Beds hos $_{\text {ho }}$ & Beds $_{I C U}$ & Patients $_{\text {hos }}$ & Patients ${ }_{I C U}$ & Days $_{\text {hos }}$ & Days $_{I C U}$ & Doctors $_{\mathrm{ICU}}$ & Nurses $_{I C U}$ \\
\hline \multicolumn{10}{|l|}{ Public } \\
\hline \multicolumn{10}{|l|}{ General } \\
\hline Tertiary & 877 & $1,246,054$ & 25,054 & $49,534,487$ & $1,052,682$ & $449,420,159$ & $7,177,717$ & 13,439 & 47,193 \\
\hline Secondary & 698 & 389,854 & 6777 & $15,164,592$ & 285,984 & $226,320,512$ & $14,097,614$ & 3991 & 11,558 \\
\hline \multicolumn{10}{|l|}{ Specialized } \\
\hline Tertiary & 153 & 111,351 & 4717 & $5,921,433$ & 206,430 & $49,887,588$ & $1,654,392$ & 2250 & 6819 \\
\hline Secondary & 21 & 3369 & 368 & 195,704 & 16,176 & $1,619,987$ & 75,098 & 156 & 379 \\
\hline \multicolumn{10}{|l|}{ Private } \\
\hline \multicolumn{10}{|l|}{ General } \\
\hline Tertiary & 41 & 39,346 & 725 & $1,389,714$ & 25,114 & $15,842,900$ & 157,202 & 320 & 1135 \\
\hline Secondary & 58 & 25,768 & 507 & 907,210 & 18,440 & $7,701,873$ & 118,324 & 273 & 815 \\
\hline Total & 1848 & $1,815,742$ & 38,148 & $73,113,140$ & $1,604,826$ & $750,793,019$ & $23,280,347$ & 20,429 & 67,899 \\
\hline
\end{tabular}

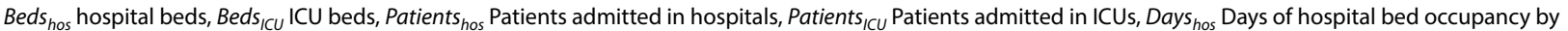
patients, Days ICU Days of ICU bed occupancy by patients, Doctors ICU ICU doctor number, Nurses ICU ICU nurse number 
Table 2 Compliance of surviving sepsis campaign (SSC) guidelines (Cssc) of different types of hospitals

\begin{tabular}{|c|c|c|c|c|c|c|c|c|c|}
\hline & $3 h C_{s s c}$ & $3 \mathrm{~h} \mathrm{C}_{\text {ssc-lac }}$ & $3 \mathrm{~h} C_{\text {ssc-cul }}$ & $3 \mathrm{~h} C_{\text {ssc-spe }}$ & $3 \mathrm{~h} \mathrm{C}_{\text {ssc-res }}$ & $6 \mathrm{~h} C_{s s c}$ & $6 \mathrm{~h} C_{\text {ssc-rep }}$ & $6 \mathrm{~h} C_{\text {ssc-vas }}$ & $6 \mathrm{~h} \mathrm{C}_{\text {ssc-CVP }}$ \\
\hline \multicolumn{10}{|l|}{ Public } \\
\hline \multicolumn{10}{|l|}{ General } \\
\hline Tertiary & 0.75 & 0.82 & 0.75 & 0.85 & 0.78 & 0.64 & 0.66 & 0.66 & 0.49 \\
\hline Secondary & 0.75 & 0.79 & 0.74 & 0.83 & 0.75 & 0.63 & 0.65 & 0.65 & 0.49 \\
\hline \multicolumn{10}{|l|}{ Specialized } \\
\hline Tertiary & 0.70 & 0.76 & 0.70 & 0.80 & 0.74 & 0.59 & 0.67 & 0.67 & 0.52 \\
\hline Secondary & 0.68 & 0.86 & 0.78 & 0.91 & 0.79 & 0.64 & 0.79 & 0.70 & 0.56 \\
\hline \multicolumn{10}{|l|}{ Private } \\
\hline \multicolumn{10}{|l|}{ General } \\
\hline Tertiary & 0.76 & 0.78 & 0.72 & 0.87 & 0.79 & 0.68 & 0.61 & 0.57 & 0.46 \\
\hline Secondary & 0.68 & 0.82 & 0.83 & 0.89 & 0.84 & 0.63 & 0.72 & 0.68 & 0.50 \\
\hline Total & 0.74 & 0.80 & 0.74 & 0.84 & 0.77 & 0.63 & 0.66 & 0.66 & 0.50 \\
\hline
\end{tabular}

SSC surviving sepsis campaign, $C_{\text {ssc }}$ compliance of SSC guidelines, $3 h C_{\text {ssc-lac }}$ completion of lactate concentration was determined, $3 h C_{s s c-c u l}$ completion of appropriate routine microbiologic cultures (including blood) be obtained before starting antimicrobial therapy, $3 h C_{\text {ssc-spe }}$ completion of empiric broad-spectrum therapy, $3 h C_{s s c-}$ res completion of resuscitation with $30 \mathrm{ml} / \mathrm{kg}$ crystal liquid, $6 \mathrm{~h} C_{\text {ssc-rep }}$ completion of repeated measurement of lactate levels in patients with initial hyperlactatemia, $6 \mathrm{~h}$ $C_{s s c-v a s}$ completion of resuscitation with vasopressor in patients with MAP $\leq 65 \mathrm{mmHg}$ after fluid resuscitation, $6 \mathrm{~h} \mathrm{C}_{\text {ssc-Cvp }}$ completion of CVP and ScvO2 were measured in patients with lactate $\geq 4 \mathrm{mmol} / \mathrm{L}$

aspects of the work in ensuring that questions related to the accuracy or integrity of any part of the work are appropriately investigated and resolved.

\section{Data collection}

In all of the participating clusters, data were obtained and entered into a web-based data entry system by a local, trained independent research coordinator. Range checks were used to check for inconsistent or out-ofrange data, prompting the user to correct or review data entries outside the predefined range. The system also provided predefined logic checks to identify errors or illogical data entries. A data quality meeting was held monthly to review all of the hospital enrollment records and registry data.

\section{Data analysis}

Statistical analysis was performed using SPSS software, version 16.0 (IBM Corp., Armonk, NY, USA). The Kolmogorov-Smirnov test was employed to check whether the data were normally distributed. The results are described as mean \pm standard deviation. Comparisons between multiple groups were analyzed by one-way analysis of variance (ANOVA), and pairwise comparisons after ANOVA were conducted using the Tukey multiple comparisons test. All of the statistical tests were two-tailed, and a $P<0.05$ was considered to be statistically significant.

\section{Results}

Correlations between the structure indicators of ICU and $\mathrm{C}_{\text {ssc }}$.

No statistically significant difference was found with respect to the proportion of ICU patients among total inpatients. There was no relationship of 3 and $6-\mathrm{h} C_{\mathrm{ssc}}$ with the proportion of ICU patients among total inpatients (Fig. 1a).

3-h $\mathrm{C}_{\mathrm{ssc}}$ in the lower, higher and highest group of the proportion of ICU in total inpatient bed occupancy was significantly higher than that in the lowest group $(P<0.05)$ (Fig. 1b). However, same phenomenon was not observed in hour- 6 bundle and each sub-index of hour-3 bundle (Fig. 1b).

In the lower, higher and highest group of the proportion of APACHE II scores $\geq 15$ in all ICU patients, 3 -h $\mathrm{C}_{\mathrm{ssc}}$ was significantly higher than that in the lowest group $(P<0.05)$ (Fig. 1c). Completion of empiric broadspectrum therapy in the lower and higher group was significantly higher than that in the lowest group $(P<0.05)$ (Fig. 1c). However, same phenomenon was not observed in hour- 6 bundle other sub-index of hour- 3 bundle and sub-index of hour- 6 bundle (Fig. 1c).

6-h $\mathrm{C}_{\mathrm{ssc}}$ in the lower, higher and highest group of the predicted mortality rate was significantly higher than that in the lowest group $(P<0.05)$ (Fig. 1d). 6-h $\mathrm{C}_{\mathrm{ssc}}$ in the higher and highest group was significantly higher than that in the lower group $(P<0.05)$ (Fig. $1 \mathrm{~d}) .6-\mathrm{h}_{\mathrm{ssc}}$ in the highest group was significantly higher than that in the higher group $(P<0.05)$ (Fig. 1d). Completion of each 

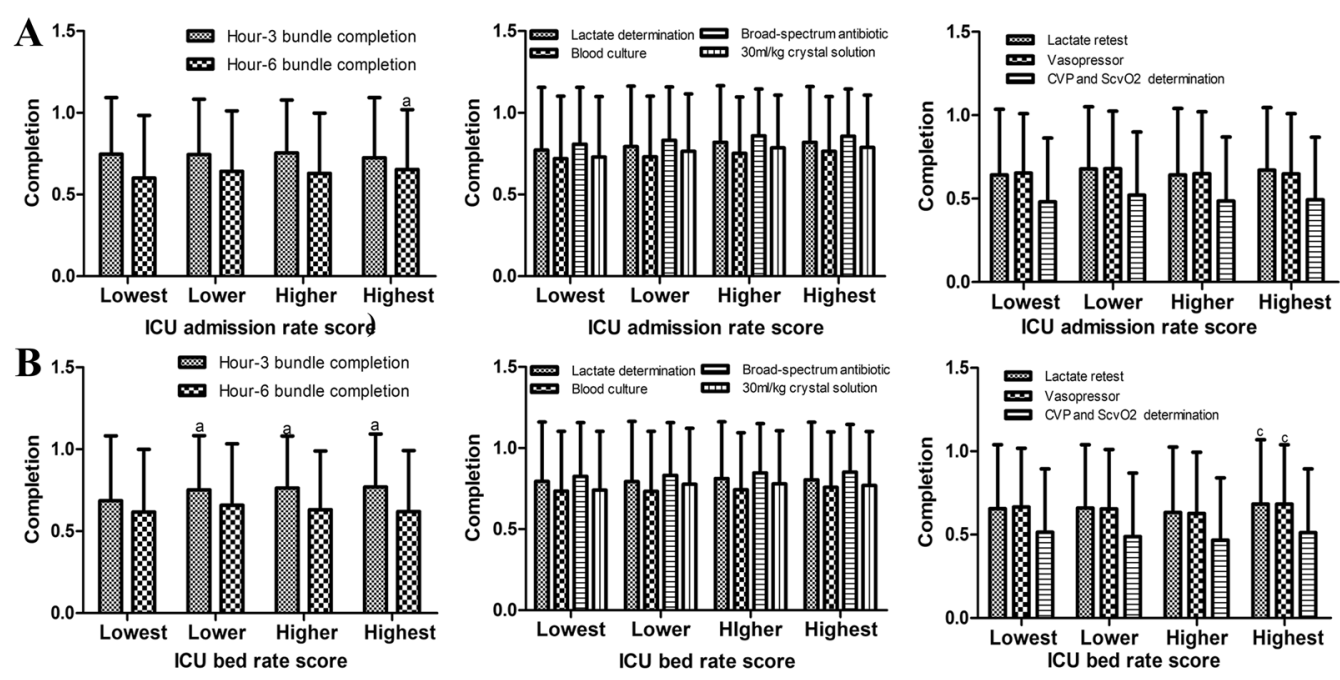

ICU admission rate score
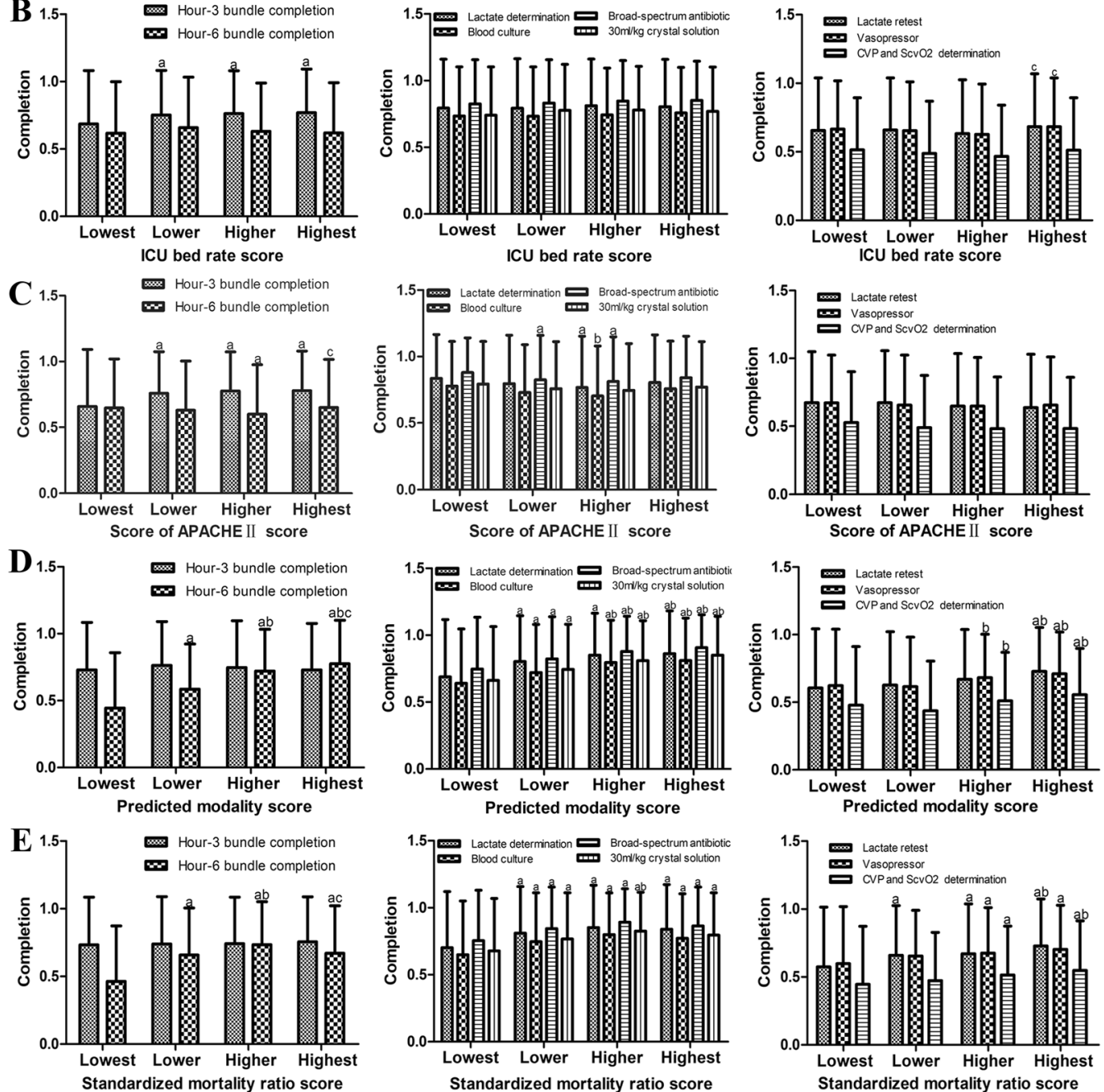

Fig. 1 Correlation between the structure indicators and compliance of surviving sepsis campaign (SSC) guidelines ( $C_{\text {ssc }}$ ). Each indicator is divided into 4 grades according to the implementation. Each 25\% from bad to good is one level, with 0, 1, 2, or 3 points. According to the scores, they are divided into the lowest group, the lower group, the higher group, and the highest group. Results are presented as mean \pm SD. $\mathbf{a}=P<0.05$ compared to the lowest group, $\mathbf{b}=P<0.05$ compared to the lower group, $\mathbf{c}=P<0.05$ compared to the higher group. DVT=deep vein thrombosis. $I C U=$ intensive care unit. ICU admission rate = the proportion of ICU patients among total inpatients, ICU bed rate = the proportion of ICU patients out of total inpatient bed occupancy, APACHE II score = the proportion of APACHE II scores $\geq 15$ in all ICU patients

sub-index of hour-3 bundle in the lower, higher and highest group was significantly higher than that in the lowest group $(P<0.05)$ (Fig. 1d). Completion of each sub-index of hour-3 bundle in the higher and highest group was significantly higher than that in the lower group except completion of lactate concentration was determined in the higher group $(P<0.05)$ (Fig. 1d). Completion of each sub-index of hour- 6 bundle in the higher and highest group was significantly higher than that in the lower group except completion of repeated measurement of 
lactate levels in patients with initial hyperlactatemia in the higher group $(P<0.05)$ (Fig. 1d). These results indicated that higher predicted mortality rate mean better $\mathrm{C}_{\mathrm{ssc}}$.

6-h $\mathrm{C}_{\text {ssc }}$ in the lower, higher and highest group of the standardized mortality ratio was significantly higher than that in the lowest group $(P<0.05)$ (Fig. 1e). 6-h $\mathrm{C}_{\mathrm{ssc}}$ in the higher group was significantly higher than that in the lower group $(P<0.05)$ (Fig. 1e). Completion of each sub-index of hour-3 bundle in the lower, higher and highest group was significantly higher than that in the lowest group $(P<0.05)$ (Fig. 1e). Completion of repeated measurement of lactate levels in patients with initial hyperlactatemia in the lower, higher and highest group was significantly higher than that in the lowest group $(P<0.05)$ (Fig. 1e). Completion of resuscitation with vasopressor in patients with $\mathrm{MAP} \leq 65 \mathrm{mmHg}$ after fluid resuscitation and completion of CVP and $\mathrm{ScvO} 2$ were measured in patients with lactate $\geq 4 \mathrm{mmol} / \mathrm{L}$ in the higher and highest group were significantly higher than that in the lowest group $(P<0.05)($ Fig. 1e). These results indicated that lower standardized mortality ratio mean better $C_{\text {ssc }}$.

\section{Discussion}

On March 15, 2012, the Ministry of Health of China approved that Peking Union Medical College Hospital establish China-NCCQC. The Quality Improvement of Critical Care Program, led by China-NCCQC, was initiated in 2015. This study is part of the above program. $C_{\text {ssc }}$ is closely related to patient prognosis [6]. At present, researches on $C_{\text {ssc }}$ mainly focus on the relationship between $C_{\text {ssc }}$ and prognosis [8,9], and there are few researches on $C_{\text {ssc }}$ itself and its influencing factors [10, 11]. How to improve $C_{\text {ssc }}$ becomes an important part of clinical practice. Wang found that the $C_{\text {ssc }}$ of emergency physicians is often hindered by the doctors' awareness and attitudes [12]. ICU structure indicators could affect the incidence of ICU-acquired infections and clinical outcomes [13]. Therefore, we designed this study to investigate the relationship between structure indicators of ICU and $C_{\text {ssc }}$ in China. In our previous study, a multifaceted $\mathrm{Q}_{\mathrm{ICU}}$ intervention was effective in improving 3 and 6-h $C_{\text {ssc }}$ in septic shock in China [14]. In this study, we found that 6-h $C_{\text {ssc }}$ was lower than 3-h $C_{\text {ssc }}$ In the subgroup, completion of empiric broad-spectrum therapy was the highest, and completion of CVP and $\mathrm{ScvO} 2$ measured in patients with lactate $\geq 4 \mathrm{mmol} / \mathrm{L}$ was the lowest, which might be why 6-h $\underline{C}_{\mathrm{ssc}}$ is lower than 3-h $C_{\text {ssc. }}$ The key to improving 6-h $C_{\mathrm{ssc}}$ and even the whole $C_{\mathrm{ssc}}$ is improving completion of CVP and $\mathrm{ScvO} 2$ measured in patients with lactate $\geq 4 \mathrm{mmol} / \mathrm{L}$. In the 3 -h subgroup, completion of appropriate routine microbiologic cultures (including blood) before starting antimicrobial therapy was the lowest. The key to improve 3-h $C_{\text {ssc }}$ is improving completion of appropriate routine microbiologic cultures (including blood) before starting antimicrobial therapy.

Specifically, 3 and 6-h $C_{\mathrm{ssc}}$ is related to predicted mortality rate, standardized mortality ratio. 3 and 6-h $C_{\mathrm{ssc}}$ was better when the predicted mortality rate was higher, and the standardized mortality ratio was lower. The higher that the predicted mortality rate is, the higher that the proportion of patients admitted to the ICU with severe diseases is. The lower that the standardized mortality ratio is, the higher that the diagnosis and treatment level in the ICU is [15-17]. The combination of the above two indicators can fully reflect the medical level of an ICU. Higher levels lead to better $C_{\text {ssc }}$.

The relationship of 3 and 6-h $C_{\text {ssc }}$ with the proportion of ICU patients among total inpatient bed occupancy, the proportion of APACHE II score $\geq 15$ in all ICU patients is uncertain and further research is needed. Interestingly, predicted mortality rate, which is closely related to APACHE II score is related to 3 and 6-h $C_{\text {ssc }}$. This phenomenon might reflect the difference in test titers between the two indicators. When examining 3 and 6-h $C_{\text {ssc }}$, predicted mortality rate might be a more effective indicator.

While the relationship of 3 and $6-\mathrm{h} C_{\text {ssc }}$ with the proportion of ICU patients among total inpatient bed occupancy is uncertain, there was no relationship of 3 and 6-h $C_{\text {ssc }}$ with the proportion of ICU patients among total inpatients. Reason for the above phenomenon may be that both the proportion of ICU patients among total inpatient bed occupancy and $C_{\mathrm{ssc}}$ are correlated with hospital treatment level, while the proportion of ICU patients among total inpatients is not correlated with hospital treatment level.

The factors confounding the study include the equipment in ICU, the transport capacity of the hospital, the cooperation of relevant departments, the technical level of medical staff and so on. For example, whether there is lactate detection equipment in the ICU, whether the field staff can send samples to the relevant testing departments in time, and whether the relevant testing departments can conduct tests immediately after receiving the samples can significantly affect the monitoring of lactate. It can be seen from our investigation that the completion of lactate concentration measurement was lower than the completion of empiric broad-spectrum therapy. The latter can be done independently in the ICU. The completion of CVP and $\mathrm{ScvO} 2$ measurement, which required the highest technical level of medical staff, was the lowest among the items of 3 and $6-\mathrm{h} C_{\text {ssc. }}$.

There are some limitations of our study. First, since only 1 year of data was included in this study, the relationships 
of the structure indicators of ICU on 3 and 6-h $C_{\text {ssc }}$ could not be analyzed continuously and dynamically. Second, those hospitals enrolled from China-NCCQC might be more motivated to improve sepsis care quality than other hospitals. Further studies will be necessary to determine the relationships of the structure indicators of ICU on 3 and 6-h $C_{\text {ssc }}$ in hospitals in China that differ in characteristics from those that participated.

\section{Conclusions}

The key to improving 6-h $C_{\mathrm{ssc}}$ and even the whole $C_{\mathrm{ssc}}$ is to improve completion of $\mathrm{CVP}$ and $\mathrm{ScvO} 2$ being measured in patients with lactate $\geq 4 \mathrm{mmol} / \mathrm{L}$. The key to improve 3-h $C_{\text {ssc }}$ is to improve completion of appropriate routine microbiologic cultures (including blood) before starting antimicrobial therapy. The factors influencing 3 and 6-h $C_{\text {ssc }}$ in China are the predicted mortality rate and the standardized mortality ratio.

\section{Abbreviations \\ SSC: Surviving sepsis campaign; $C_{\text {ssc }}$ : Compliance with the surviving sepsis campaign guidelines; ICU: Intensive care unit; China-NCCQC: China National Critical Care Quality Control Center; MAP: Mean arterial pressure; CVP: Central venous pressure; ScvO2: Central venous oxygen saturation.}

\section{Supplementary Information}

The online version contains supplementary material available at https://doi. org/10.1186/s40001-021-00498-7.

Additional file 1: Basic information of hospitals in different provinces and cities. $a=$ Hospitals, $b=$ Beds, $c=I C U$ beds, $d=$ Patients admitted in hospitals, $\mathrm{e}=$ Patients admitted in ICUs, $\mathrm{f}=$ Days of hospital bed occupancy by patients, $g=$ Days of ICU bed occupancy by patients, $h=I C U$ doctor number, $\mathrm{i}=\mathrm{ICU}$ nurse number.

Additional file 2: Figure S1 3 hours SSC bundles compliance rate (\%) of hospitals in different provinces and cities. Figure $\mathbf{S} \mathbf{2}$ Compliance rate of lactate concentration was determined in different provinces and cities. Figure $\mathbf{S 3}$. Compliance rate of microbiologic cultures before antimicrobial therapy in different provinces and cities. Figure $\mathbf{S 4}$. Compliance rate of empiric broad-spectrum therapy in different provinces and cities. Figure S5. Compliance rate of resuscitation with $30 \mathrm{ml} / \mathrm{kg}$ crystal liquid in different provinces and cities.

Additional file 3: Figure 1.6 hours SSC bundles compliance rate (\%) of hospitals in different provinces and cities. Figure $\mathbf{2}$. Compliance rate of retest of lactate levels in patients with initial hyperlactatemia in different provinces and cities. Figure 3. Compliance rate of resuscitation with vasopressor if MAP $\leq 65 \mathrm{mmHg}$ after fluid resuscitation in different provinces and cities. Figure 4. Compliance rate of CVP and $\mathrm{S} C \mathrm{NO} 2$ were measured in patients with lactate $\geq 4 \mathrm{mmol} / \mathrm{L}$ in different provinces and cities.

\section{Acknowledgements}

We thank Wei Huang for her technical support and editorial assistance. In all of the participating clusters, data were obtained and entered into a web-based data entry system by a local, trained independent research coordinator who was not involved in the care of patients and who received compensation for this trial.

Monitored email of China National Critical Care Quality Control Center group:china_nccqc@163.com.

\section{Authors' contributions}

$X Z, D W L \& Y L$ conceived and designed the experiments. LW, XDM, HWH, LXS, YHG \& GLS analyzed the data, and wrote the manuscript. AND, LW, XDM \& $X Z$ edit and revised the manuscript. All authors read and approved the final manuscript.

\section{Funding}

This research was supported by National Key R\&D Program of China (grant number 2020YFC0861000) and National Natural Science Foundation of China (No. 81801901).

\section{Availability of data and materials}

The datasets supporting the conclusions of this article are included within the article and its additional files.

\section{Declarations}

Ethics approval and consent to participate

The study was conducted in accordance with the Declaration of Helsinki (as revised in 2013). The trial protocol was approved by the Central Institutional Review Board at Peking Union Medical College Hospital (NO.: S-K1297) and individual consent for this retrospective analysis was waived. The authors are accountable for all aspects of the work in ensuring that questions related to the accuracy or integrity of any part of the work are appropriately investigated and resolved.

\section{Consent for publication}

All authors have seen and agreed with the contents of the manuscript, and the manuscript has been submitted solely to this journal and is not published, in press, or submitted elsewhere.

\section{Competing interests}

The authors declare that they have no competing interests.

\section{Author details}

${ }^{1}$ Department of Critical Care Medicine, Peking Union Medical College and Chinese Academy of Medical Sciences, Peking Union Medical College Hospital, Beijing 100730, China. ${ }^{2}$ State Key Laboratory of Complex Severe and Rare Diseases, Peking Union Medical College Hospital, Chinese Academy of Medical Science and Peking Union Medical College, Beijing 100000, China. ${ }^{3}$ Department of Medical Administration, National Health Commission of the People's Republic of China, Beijing 100000, China. ${ }^{4}$ Department of Epidemiology and Biostatistics, Institute of Basic Medicine Sciences, Chinese Academy of Medical Sciences (CAMS) \&School of Basic Medicine, Peking Union Medical College, Beijing 100000, China.

Received: 24 November 2020 Accepted: 5 March 2021

Published online: 19 March 2021

\section{References}

1. Singer M, Deutschman CS, Seymour CW, et al. The Third international consensus definitions for sepsis and septic shock (Sepsis-3). JAMA. 2016:315:801-10.

2. Vieillard-Baron A, Caille V, Charron C, Belliard G, Page B, Jardin F. Actual incidence of global left ventricular hypokinesia in adult septic shock. Crit Care Med. 2008;36:1701-6.

3. Stevenson EK, Rubenstein AR, Radin GT, Wiener RS, Walkey AJ. Two decades of mortality trends among patients with severe sepsis: a comparative meta-analysis*. Crit Care Med. 2014;42:625-31.

4. Ani C, Farshidpanah S, Bellinghausen Stewart A, Nguyen HB. Variations in organism-specific severe sepsis mortality in the United States: 1999-2008. Crit Care Med. 2015;43:65-77.

5. Herran-Monge R, Muriel-Bombin A, Garcia-Garcia MM, et al. Epidemiology and changes in mortality of sepsis after the implementation of surviving sepsis campaign guidelines. J Intens Care Med. 2017885066617711882.

6. Levy MM, Rhodes A, Phillips GS, et al. Surviving Sepsis Campaign: association between performance metrics and outcomes in a 7.5-year study. Critical Care Med. 2015;43:3-12. 
7. Raj SK, Panda PK, Wig N, Agarwal P, Pandey RM. Compliance with 6 h-sepsis resuscitation bundle of surviving sepsis campaign before and after resident physicians' training: a quality improvement interventional study among indian patients. J Emerg Trauma Shock. 2019;12:3-9.

8. Workman JK, Ames SG, Reeder RW, et al. Treatment of pediatric septic shock with the surviving sepsis campaign guidelines and PICU patient outcomes. Pediatric Criti Care Med. 2016;17:e451-8.

9. Rhodes A, Phillips G, Beale R, et al. The Surviving Sepsis Campaign bundles and outcome: results from the International Multicentre Prevalence Study on Sepsis (the IMPreSS study). Intensive Care Med. 2015;41:1620-8.

10. Levy MM, Dellinger RP, Townsend SR, et al. The Surviving Sepsis Campaign: results of an international guideline-based performance improvement program targeting severe sepsis. Crit Care Med. 2010;38:367-74.

11. Mukherjee V, Evans L. Implementation of the surviving sepsis campaign guidelines. Curr Opin Crit Care. 2017;23:412-6.

12. Wang Z, Xiong Y, Schorr C, Dellinger RP. Impact of sepsis bundle strategy on outcomes of patients suffering from severe sepsis and septic shock in china. J Emerg Med. 2013;44:735-41.

13. Garrouste-Orgeas M, Soufir L, Tabah A, et al. A multifaceted program for improving quality of care in intensive care units: IATROREF study. Crit Care Med. 2012:40:468-76.
14. He H, Ma X, Su L, et al. Effects of a national quality improvement program on ICUs in China: a controlled pre-post cohort study in 586 hospitals. Crit Care. 2020;24:73.

15. Manaseki-Holland S, Lilford RJ, Te AP, et al. Ranking hospitals based on preventable hospital death rates: a systematic review with implications for both direct measurement and indirect measurement through standardized mortality rates. Milbank Q. 2019;97:228-84.

16. Kashyap R, Singh TD, Rayes H, et al. Association of septic shock definitions and standardized mortality ratio in a contemporary cohort of critically ill patients. J Crit Care. 2019;50:269-74.

17. Ngantcha M, Le-Pogam MA, Calmus S, et al. Hospital quality measures: are process indicators associated with hospital standardized mortality ratios in French acute care hospitals? BMC Health Serv Res. 2017;17:578.

\section{Publisher's Note}

Springer Nature remains neutral with regard to jurisdictional claims in published maps and institutional affiliations.
Ready to submit your research? Choose BMC and benefit from:

- fast, convenient online submission

- thorough peer review by experienced researchers in your field

- rapid publication on acceptance

- support for research data, including large and complex data types

- gold Open Access which fosters wider collaboration and increased citations

- maximum visibility for your research: over $100 \mathrm{M}$ website views per year

At BMC, research is always in progress.

Learn more biomedcentral.com/submissions 